\title{
Metodología para realizar mini-vídeos accesibles y multilingües para formación universitaria A methodology to create accessible \& multiligual mini-videos to learning higher education
}

\author{
Lina García-Cabrera $^{1}$, Ildefonso Ruano-Ruano ${ }^{2}$, José Ramón Balsas-Almagro ${ }^{1}$, Raquel Fuentes-Martínez ${ }^{3}$ \\ E-mail: lina@ujaen.es, alonso@ujaen.es,jrbalsas@ujaen.es, rfuentes@ujaen.es \\ ${ }^{1}$ Departamento de Informática \\ Universidad de Jaén \\ Jaén, España \\ ${ }^{2}$ Departamento de Ingeniería de \\ Telecomunicación \\ Universidad de Jaén \\ Jaén, España \\ ${ }^{3}$ Departamento de Filología Inglesa \\ Universidad de Jaén \\ Jaén, España
}

\begin{abstract}
Resumen- La formación a la largo de la vida es necesaria en cualquier institución o empresa. La autoformación mediante minivídeos es una opción ágil y efectiva. Sin embargo, la elaboración de estos materiales digitales supone un costo en recursos, tiempo, esfuerzo y dinero que puede ser inasumible. Asimismo, estos recursos multimedia suelen incumplir la Directiva Europea de Accesibilidad Digital. En este trabajo se presenta una metodología para la elaboración de mini-vídeos formativos de bajo coste, accesibles y multilingües. Entendiendo como mini-videos a aquellos que tienen una duración de entre 5 y 10 minutos aproximadamente. De igual modo, se presenta un proyecto en desarrollo en el que se aplicará dicha metodología en la formación que realiza la Biblioteca de la Universidad de Jaén. Esta praxis permitirá refinar la metodología, evaluar su eficacia en función del coste, y en su caso, proponer un proceso sistemático de elaboración de mini-vídeos que se pueda integrar fácilmente en la gobernanza de Tecnología de la Información (TI) de cualquier institución, organismo o empresa.
\end{abstract}

\section{Palabras clave: Educación Universitaria, Aprendizaje a lo largo de la vida, recursos multimedia accesibles, elearning modular, mini- vídeos, accesibilidad, multilingüismo}

\begin{abstract}
Lifelong learning is necessary at any institution or company. Self-training using mini-videos is an agile and effective option. However, the development of digital material needs resources: time, effort and money that could be unaffordable. In addition, these multimedia resources usually fail to comply the European Directive on Digital Accessibility. This paper presents a methodology for the development of low cost and accessible training mini-videos with multilingual support. It is understood that a minivideo is a video whose duration is approximately 5-10 minutes. Also, it presents the project in which this methodology and techniques are being applied for the training carried out by the Service of Library at the University of Jaén. This proposal will allow to refine and assess effectiveness and cost of current training. Finally, the proposal aims to define a systematic process of developing mini-videos that can be easily integrated into the Information Technology (IT) governance of any institution, organization or company.
\end{abstract}

Keywords: Higher Education, lifelong learning, accessible multimedia resource, elearning modules, mini-videos, accessibility, multilingualist

\section{INTRODUCCIÓN}

El Espacio Europeo de Educación Superior (EEES) supone un cambio metodológico en la educación superior que se basa en un modelo pedagógico fundamentado en el aprendizaje dirigido por el docente y continuado a lo largo de la vida (Fejes \& Nicoll, 2008).

En este contexto la Biblioteca de la Universidad de Jaén (UJA) propone un programa de alfabetización informacional dirigido a sus usuarios teniendo como referente las premisas siguientes:

- La adquisición y dominio de las competencias informacionales facilitan que los titulados universitarios puedan enfrentarse con mayores garantías de éxito a la innovación de los campos científicos y profesionales en los que desarrollen su actividad laboral futura. Les ayudan, también, a resolver problemas de todo tipo y a comprender mejor el entorno en el que viven.

- El dominio de las competencias y habilidades implicadas en la formación informacional posibilitan a los graduados universitarios poder seguir aprendiendo por sí mismos a lo largo de toda la vida.

- Las competencias informacionales favorecen los procesos de aprendizaje constructivistas del conocimiento $\mathrm{y}$, en consecuencia, permiten la implementación de modelos didácticos coherentes con el Sistema Europeo de Transferencia de Créditos (ECTS).

El e-learning (CRUETIC, 2015; Marketdata Enterprises, 2011) y, en particular, su variedad mixta o b-learning (Heinze\&Procter, 2004) es una de las maneras más eficaces para la autoformación y el aprendizaje a lo largo de la vida (García-Cabrera, 2013, 2013a, 2013b). En este sentido, los cursos de formación complementarios o de especialización requieren de un tiempo y unos horarios imposibles para la mayor parte de la comunidad universitaria.

Está demostrado que el uso de mini-tutoriales de vídeo en estos cursos permite una formación más flexible y autosuficiente (Letón, Durban, D'Auria, \& Lee, 2009, Kay, 
2012), ya que combinan tres características muy necesarias en cualquier proceso de aprendizaje, como son la proximidad de una presentación cara-a-cara, su finalidad práctica y estar centrados en un tema muy concreto (Valderrama, Sánchez, \& Urrejola, 2009). Las principales ventajas de los mini-vídeos para los estudiantes son la posibilidad de elegir el momento y el lugar de aprendizaje, así como la de acceder a los mismos tantas veces como deseen. Por otro lado, los mini-vídeos tienen una gran cantidad de ventajas para los profesores como la posibilidad de ser utilizados por diferentes sujetos o el reforzamiento de algunos conceptos o ejemplos para los estudiantes. Por tanto, los mini-vídeos pueden ser considerados como píldoras de conocimiento (Sánchez, Cancela, Maceiras \& Urrejola 2010) (Bengochea Martínez, 2011), ya que son contenidos de e-learning diseñados para proporcionar una sesión informativa, de repaso o de formación sobre un tema específico en unos 10 minutos o menos.

A veces los mini-vídeos consisten simplemente en la grabación de conferencias enteras que son vistas de forma pasiva (Thaphagan et al., 2010). Sin embargo, los mini tutoriales en vídeo son recursos especialmente diseñados para la enseñanza de un tema específico para un sujeto.

Estos mini-vídeos, en contraste con otros recursos didácticos, tienen algunos problemas de accesibilidad (Bengochea\&Medina, 2013, Boldyreff et al., 2001). Cuando diseñamos vídeos para un grupo de estudiantes, es necesario tener en cuenta algunos aspectos de accesibilidad que permitan utilizarlos a las personas con algún tipo de diversidad funcional.

Elementos como los subtítulos, la transcripción o la posibilidad de elegir entre diferentes idiomas son esenciales para destacar un mini-vídeo como un bueno. Además, cuando se elabora un vídeo, para que luego sea subtitulado, se realiza un mayor esfuerzo en enseñar lo esencial, en usar un lenguaje más claro y en cuidar la vocalización/dicción. Siguiendo las recomendaciones de (Bengochea \& Budia, 2012), y los resultados de anteriores proyectos de innovación (BalsasAlmagro, 2016), proponemos algunas características al realizar un buen mini-vídeo:

- La duración del vídeo debe ser de aproximadamente de 5 a 10 minutos.

- Se debe utilizar recursos ágiles y mínimos.

- Es necesario tener una cierta calidad de vídeo y sonido usando un sistema de grabación de calidad.

- Es importante utilizar los formatos estándar del W3C.

- Es necesario sincronizar imágenes, texto y sonido.

- Usar un lenguaje sencillo y claro, en voz activa, si es posible (tratando de evitar formas pasivas).

- Proporcionar conceptos esenciales, eliminando las partes no relevantes.

Sin embargo, la producción de mini-vídeos implica dedicar tiempo y a menudo la utilización de una gran cantidad de herramientas complejas. Por otra parte, los formadores no suelen conocer el proceso teórico o práctico para elaborarlos.

En este trabajo se parte de la experiencia de varios proyectos de innovación docente centrados en la enseñanza virtual\&blended (Garrison \& Vaughan, 2008), en la elaboración de material/recursos de aprendizaje y el desarrollado de un proceso sistemático en el que se usan herramientas libres para hacer mini-vídeos de una manera regular. El resultado es un proceso para elaborar mini-vídeos accesibles (subtitulados y transcritos), con soporte internacional (multilingües) y de bajo costo, tanto en tiempo como en dinero. Se ha propuesto un proceso flexible y sencillo en el que se hace uso de herramientas de Google App para que el proceso de transcripción, subtitulación y traducción sea lo más rápido posible. Las Google Apps son aplicaciones, generalmente gratuitas, que proporciona la empresa Google, son accesibles desde cualquier navegador moderno y permiten la edición de diferentes tipos de contenidos por parte de varios usuarios que trabajan en colaboración (Balsas-Almagro, 2016).

Como resultado, se ha elaborado una metodología, que presentamos en esta contribución, que se pretende aplicar en instituciones y/o empresas que requieran formación específica a lo largo de la vida. El objetivo es fomentar la realización masiva de mini-vídeos de bajo coste en recursos, tiempo y dinero.

Para comprobar la viabilidad de esta metodología, en este proyecto se cuenta con la participación, colaboración y los recursos del Servicio de Biblioteca de la Universidad de Jaén. Desde hace varios años, el personal de Biblioteca, como se ha mencionado antes, viene impartiendo los cursos de Alfabetización Informacional (ALFIN) a toda la comunidad universitaria, están organizados en cuatro itinerarios formativos dirigidos a alumnos de Grado, estudiantes de postgrado, personal docente e investigador y personal de administración y servicios. En este sentido, el personal de Biblioteca ha sido muy activo en esta formación y ha desarrollado multitud de tutoriales complementarios.

En este proyecto de innovación docente se pretende aunar esfuerzos y hacer transferencia de las buenas prácticas desarrolladas por el equipo docente involucrado en numerosos proyectos de innovación docente (García-Cabrera, 2009, 2010, 2012, 2012a). En particular, se tratará de asesorar y participar junto al personal de biblioteca en la elaboración de los vídeos de calidad (Pérez-Perez, 2011), subtitulándolos en varios idiomas, siguiendo buenas prácticas y la metodología desarrollada por el equipo docente. De igual modo, se busca constatar la eficacia del proceso para luego divulgarlo al resto de la comunidad universitaria y extrapolarlo a otras instituciones o empresas que requieran formación a lo largo de la vida.

\section{CONTEXTO}

Este proyecto tiene como objetivo general fomentar la formación mediante mini-vídeos y, para ello, conseguir que el proceso de realización sea de calidad y eficiente. La idea es ofrecer un proceso fácil y cooperativo, en el que algunas de las fases del proceso puedan ser externalizadas si es necesario.

El objetivo principal es enriquecer y mejorar el alcance de la formación ALFIN para ofrecer mini-vídeos de calidad, accesibles, subtitulados, transcritos y traducidos al inglés. Esta colaboración permitirá mejorar la formación y hacerla más accesible (Krug, 2013, Loranger, 2014) e internacional pudiéndose beneficiar al colectivo con alguna discapacidad auditiva (se seguirán las pautas de accesibilidad web desarrolladas por la W3C, las guías WCAG 2.0 Nivel de conformidad A y AA) y también a los estudiantes de Erasmus. 
Adicionalmente, se desea constatar la viabilidad de la metodología sistemática propuesta para todo el proceso de elaboración, transcripción, subtitulación y traducción de minivídeos formativos. Su puesta en práctica nos permitirá detectar y subsanar posibles problemas e incidencias. De igual modo, se podrá medir la eficacia en tiempo y coste de dicho proceso. Para ello se realizarán estudios sobre:

- La satisfacción de las personas que aplican el proceso propuesto.

- La satisfacción y aprovechamiento del alumnado que ha usado los vídeos.

- La valoración del esfuerzo en la realización de los minivídeos.

Asimismo, se elaborará una serie de documentos en los que serán recogidos el propio proceso estándar de elaboración, así como las buenas prácticas para todas y cada una de sus fases (preparación, grabación de vídeo, subtitulado, la internacionalización y la validación) y cuestiones técnicas relacionadas con las herramientas software utilizadas en el proceso.

Finalmente, se divulgará esta propuesta y la experiencia de su aplicación a un caso real al resto del profesorado o personal involucrado en tareas de formación para que incorporen esta nueva modalidad en sus cursos.

\section{DESCRIPCIÓN}

Para la realización/selección de los mini vídeos formativos se aplicarán las siguientes buenas prácticas:

- Ajustar la duración del vídeo, debe ser de 5 a 10 minutos aproximadamente.

- Utilizar recursos ágiles y mínimos.

- Asegurar la calidad de A/V (Audio y Vídeo), es necesario tener una calidad de vídeo y sonido usando un sonido de alta calidad.

- Usar formatos estandarizados, es importante utilizar los formatos estándar del W3C.

- Sincronizar correctamente imágenes, texto y sonido.

- Usar un lenguaje sencillo y claro, en voz activa, si es posible.

- Proporcionar conceptos esenciales, eliminando las partes no esenciales.

Utilizaremos como metodología para la realización de minivídeos accesibles y multilingües nuestro propia propuesta que se caracteriza por ser:

- Abierta a todos los autores. Todos los autores deben ser capaces de seguir la metodología y las herramientas que se proponen en este trabajo sin la necesidad de tener conocimientos técnicos.

- Proceso de corta duración. El proceso no debe consumir mucho tiempo.

- Gratuito o de bajo costo. Las herramientas que se utilizan no suponen un alto coste. Es preferible utilizar herramientas gratuitas tipo freeware y de código abierto, open-source.

- Autónomo. Todo el proceso se puede realizar de manera autónoma por un solo autor.
- Cooperativo. En el caso de que varias personas quieren realizar el proceso, las herramientas permiten el trabajo cooperativo.

- Entorno heterogéneo. Los autores pueden trabajar desde cualquier sistema operativo.

- Ubicuo. Un autor puede llevar a cabo el proceso desde cualquier lugar y en cualquier momento.

- Externalizable. Las diferentes partes del proceso pueden ser subcontratados si es necesario.

Un mini-vídeo de aprendizaje puede realizarse grabando el audio "en vivo", en el mismo momento en el que se graba la imagen, o añadiendo el audio más tarde realizando una narración de los contenidos. En el segundo caso se requiere una edición del mismo con un programa adecuado. En ambos casos, las fases son la preparación, grabación de vídeo/audio, subtitulado, la internacionalización y la validación. La primera y última fase están claras, pero las otras pueden realizarse en un orden diferente o agruparlas y llevarse a cabo al mismo tiempo. La Figura 1 muestra un diagrama de flujo de las fases más comunes que se pueden presentar en el proceso de creación de mini-vídeos.

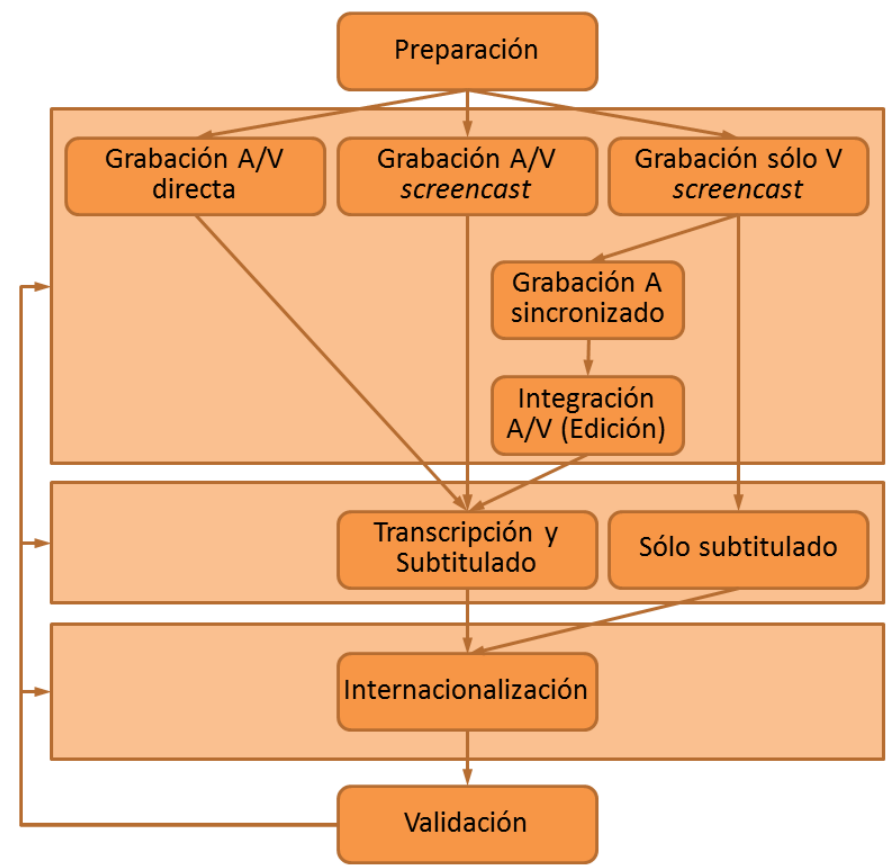

Figura 1. Fases más comunes al realizar mini-vídeos.

\section{A. Preparación}

La preparación es la primera y la fase más importante. Lo primero es realizar una buena selección de un tema autocontenido. Se debe dejar muy claro cuáles son los objetivos de aprendizaje, cómo se enseñan, qué resultados se esperan y en qué orden se presentan (no más de 10 minutos, mucho mejor si es en torno a 5 minutos). Es primordial elaborar un borrador o guión fácil de entender. Se aconseja usar oraciones simples, un vocabulario y expresiones cortas, evitar la jerga o los acrónimos. Además, usar un lenguaje estándar, activo y claro, transmitiendo información que esté basada en hechos. Trabajando de esta manera, no sólo aumenta la accesibilidad y comprensión, sino que también hace más fácil las fases de traducción y subtitulado. Durante este proceso, los autores deben ensayar al menos una o dos veces con el fin de corregir y ajustar el discurso y el tempo. 


\section{B. Grabación del Vídeo/Audio}

La grabación de vídeo se puede hacer de diferentes maneras: la grabación directa desde la cámara en el que se obtiene tanto el vídeo como el audio o desde un programa de captura de pantalla del ordenador (screencast), en cuyo caso se puede hacer grabando sólo el vídeo o el vídeo y el audio. Independientemente de la fuente, lo fundamental es la calidad del vídeo, tanto en imagen como en sonido cuando exista. La dicción, vocalización y el mantener un ritmo del discurso adecuado facilita el seguimiento y, por tanto, el aprendizaje. En algunos casos la grabación del audio se puede hacer después de la del vídeo, esto requiere el uso posteriormente de un programa de edición de vídeo que permita incorporar el audio de forma sincronizada. Existen múltiples aplicaciones con las que se pueden hacer las grabaciones, cuando se usa una cámara web es conveniente utilizar el software propio que se suele incluir con la misma. Para grabaciones tipo screencast, la oferta de software es muy variada. Entre los más utilizados se puede destacar Quicktime, Camtasia y Screenflow (MacLeod, 2017). Sin embargo, sólo Quicktime es un software libre que además se debe usar en sistemas Mac, los otros son de pago. Entre las aplicaciones de software libre, los autores del presente trabajo han podido constatar la eficacia de, al menos, el programa CamStudio, el cual ha sido utilizado por los mismos para la creación de varios minivídeos. Otra aplicación libre, que además es de código abierto, es Open Broadcast Software (OBS), además es multiplataforma (Linux, Mac y Windows), presenta facilidad de uso y versatilidad a la hora de incluir diferentes fuentes simultáneas en la grabación (pantalla, ventanas o diferentes cámaras) y permitir generar contenidos en diferentes formatos de almacenamiento.

\section{Subtitulado/Transcripción}

En la fase de subtitulado, la voz del vídeo debe ser transcrita y sincronizada. Los subtítulos se pueden crear a partir de la transcripción mediante la ruptura de texto en segmentos más pequeños llamados marcos de subtítulos, y luego sincronizarlos con el vídeo para que cada trama de subtítulo se muestre en el momento oportuno. Respecto al software de subtitulado, se evaluaron varias herramientas libres como Aegisub (código abierto y multiplataforma) o Subtitle Edit gratuita para Windows. Aunque ambas son fáciles de utilizar para el proceso de subtitulado, ninguna permite el trabajo colaborativo ni la automatización del proceso de traducción, por lo que se descartaron al no encajar en las premisas específicas de la metodología propuesta.

\section{Internacionalización}

La fase de internacionalización consiste en la traducción y, tal vez, el doblaje a varios idiomas. El texto, obtenido de la fase de transcripción, se traduce a varios idiomas para que los espectadores potenciales puedan seleccionarlos. Sin embargo, estos subtítulos traducidos podrían necesitar una sincronización individual.

\section{E. Validación}

Por último, la fase de validación comprueba la calidad de vídeo y sonido, posibles errores, una buena sincronización, y otras características que determinan la calidad del producto final. En caso de ser necesario (calidad inferior al nivel definido) se deberá volver a algunas de las fases anteriores para subsanar errores e intentar conseguir un producto mejor.
Herramientas software. El proceso de la transcripción y traducción de mini-vídeos está semiautomatizado por herramientas software de fácil uso, bajo coste y que fomenten el trabajo colaborativo. El equipo docente ha realizado una investigación y valoración de las herramientas y se ha inclinado por dos herramientas de Google Apps Suite: YouTube, para la transcripción e incluso como herramienta de traducción semiautomática de los vídeos; y Google Translation Toolkit, GTT, como entorno de traducción de subtítulos colaborativa y semiautomática.

Para la coordinación del grupo de trabajo de este proyecto de innovación se está utilizando la plataforma de docencia virtual de la Universidad de Jaén. Asimismo, se ha creado un grupo de trabajo virtual que favorece la creación colaborativa y compartida para todos los materiales de la propuesta. También se utilizan aplicaciones web 2.0 como Google drive, Skype, Hangouts de Google+.

\section{RESUltados}

Se han realizado varias pruebas en las que los autores de mini-vídeos han aplicado el proceso de transcripción y traducción de mini-vídeos. La transcripción y sincronización, en vídeos con buen sonido y una locución adecuada, se puede realizar de forma automática con buenos resultados. La traducción automática ayuda a reducir el proceso global, pero necesita de una revisión adicional para corregir traducciones literales y descontextualizadas. Para vídeos en torno a 15 minutos, aplicar el proceso completo (transcripción más traducción) supone unos 90 minutos, esto es, unos 5 minutos de trabajo por minuto de vídeo.

Estas pruebas piloto de aplicación nos han permitido proponer la metodología que se presenta en este trabajo y la sistematización de flujo de trabajo. Al mismo tiempo, se ha confeccionado un curso de formación para formar en la aplicación de este flujo de trabajo, en este caso, al personal del Servicio de Biblioteca.

Al finalizar este proyecto se esperan resultados docentes tanto para formadores y autores (esperando que el proceso facilite y haga más eficiente la producción de mini-vídeos) como para los estudiantes (esperando un aprendizaje más completo y eficiente) $\mathrm{y}$, también, de investigación y transferencia del proceso y su aplicación real.

- Se proporcionará toda la información que explica cómo elaborar mini-vídeos para cursos de $b$-learning a la carta y se compartirá con el personal de Biblioteca de la Universidad de Jaén.

- Se elaborarán guías de buenas prácticas para elaboración de mini-vídeos formativos, internacionales y accesibles, siguiendo las pautas de accesibilidad web desarrolladas por la W3C, las guías WCAG 2.0 Nivel de conformidad A y AA.

- Se desarrollarán recursos multimedia en forma de minivídeos y screencasts que hagan más flexible y eficiente el aprendizaje de cursos b-learning, en particular para los cursos ALFIN impartidos por el personal del Servicio de Biblioteca de la Universidad de Jaén.

- Se elaborarán transcripciones, subtitulaciones y traducciones para cada uno de estos mini-vídeos, teniendo especial cuidado en cuanto a la calidad del 
sonido y la sincronización de la imagen, sonido y subtítulos.

- Se realizará un estudio sobre el grado de satisfacción de los autores con respecto al proceso semi-automático de transcripción, subtitulado y traducción.

- Se realizará un estudio sobre la repercusión de estos recursos multimedia a la hora de facilitar la asimilación de los contenidos, de la accesibilidad de los mismos y de la valoración que reciban por parte del alumnado.

\section{CONCLUSIONES}

En esta propuesta se presenta una metodología para la transcripción, subtitulación y traducción de mini-vídeos para hacerlos accesibles. El objetivo es proporcionar un flujo de trabajo de bajo coste, fácil y eficiente. Esta metodología describe dicho flujo de trabajo que no requiere conocimientos técnicos, que utiliza herramientas de bajo coste, que supone poco tiempo de elaboración y facilita la colaboración.

De igual modo, describe un proyecto de innovación docente que pretende aplicar de forma generalizada dicha metodología en vídeos de formación universitaria para estudiantes locales y de Erasmus. Este caso de prueba real con autores no técnicos refinará y mejorará el proceso de aplicación. La evaluación de su eficacia y costo permitirá proponer un proceso sistemático de elaboración de mini-vídeos que se pueda integrar fácilmente en la gobernanza TI de cualquier institución, organismo o empresa que realice formación a lo largo de la vida.

\section{AGRADECIMIENTOS}

Este trabajo forma parte del proyecto de innovación docente PID53_201617 (2017-2019), financiado por el Secretariado de Enseñanzas de Grado e Innovación de la Universidad de Jaén.

\section{REFERENCIAS}

Balsas-Almagro J.R., Ruano-Ruano I., García-Cabrera L., Ortega-Tudela J.M. \& Fuentes-Martínez R. (2016). Creating Self Learning, Accessible \& Low-Cost MiniVideos(OP_085) END 2016 International Conference on Education and New Developments, 231235. 12-14 JuneLjubljana, Slovenia.

Bengochea Martínez, L. (2011). Píldoras formativas audiovisuales para el aprendizaje de programación avanzada. Proceedings of Jornadas de Enseñanza Universitaria de la Informática.

Bengochea Martínez, L. \& Budia, F. (2012). Subtitled video tutorials, an accessible teaching material. Journal of Accessibility and Design for All (CC) JACCES, 2 (2), 155-164.

Bengochea, L. y Medina, J.A. (2013). El papel de los videotutoriales accesibles en el aprendizaje del futuro. Proceedings of $\mathrm{V}$ Congreso Internacional ATICA. Huancayo, Perú. Retrieved from: www.esvial.org/wpcontent/files/Videotutoriales_BengocheaMedina.pdf

Boldyreff, C., Burd, E., Donkin, J., \& Marshall, S. (2001). The case for the use of plain English to increase web accessibility. In Web Site Evolution. Proceedings of 3rd International Workshop on IEEE, 42-48.
CRUETIC (2015). UNIVERSITIC: Descripción, gestión y gobierno de las TI en el sistema universitario español Retrieved

from: http://www.crue.org/Documentos\%20compartidos/Publi caciones/Universitic/UNIVERSITIC_2015.pdf

Fejes, A., Nicoll, K. (2008). Foucault and lifelong learning: Governing the subject. London: Routledge.

Garrison, D. R., Vaughan, N. (2008). Blended learning in higher education. San Francisco: Jossey-Bass.

Garcia-Cabrera, L.; Sanchez, P.J.; Martinez, L. Good practices in e-learning to improve the success in higher education (2009). International Conference on Engineering and Mathematics, Bilbao, Spain; 06/2009.

García-Cabrera, L.; Ortega-Tudela, J.M.; Peña-Hita, M.A.; Ruano-Ruano, I. \& Ortiz, A.M. (2010). The quality of virtual learning: The study guide importance. Pixel-Bit. Revista de medios y comunicación, 37. Retrieved from: http://www.sav.us.es/pixelbit/pixelbit/articulos/n37/7.ht $\mathrm{ml}$

García-Cabrera, L.; Ortega-Tudela, J.M.; Balsas-Almagro, J.R.; Ruano-Ruano, I.; Peña-Hita, M.A.; CuevasMartínez, J.C. (2012). New Assessment Tools in Learning Management Systems Edited by Pixel International Conference The Future of Education Florence, Italy 7-8 June 2012 Simonelli Editore University Press.

García-Cabrera, L., Ruano-Ruano, I., \& Balsas-Almagro, J. R. (2012a). Curso de formación para profesorado universitario en modalidad de b-learning sobre manejo avanzado de un entorno LMS. I Jornadas Internacionales de Innovación Docente Universitaria en entornos de aprendizaje enriquecidos. UNED, Madrid, pp. 306-308. ISBN: 84-695- 8245-3.

García-Cabrera, Lina Guadalupe; Balsas-Almagro, José Ramón; Ruano-Ruano, Ildefonso (2013). A B-Learning Tailor-Made Course On Ilias Advanced Resources For Faculty; 12th International Ilias Conference. Múnich, Alemania 29 y 30 August 2013

García-Cabrera, Lina Guadalupe; Balsas-Almagro, José Ramón; Ruano-Ruano, Ildefonso (2013a) Lifelong Learning To Academic Staff By Means Of Blended Learning \& Tailor-Made Courses;. en: 6th International Guide Conference. 3 y 4 October2013. Atenas, Grecia

García-Cabrera, L., Ruano-Ruano, I., \& Balsas-Almagro, J.R. (2013b). A b-learning methodology case for faculty at high education. Journal of Cases on Information Technology (JCIT), 15(1), 19-35.

Heinze, A., C. Procter (2004). Reflections on the Use of Blended Learning. Education in a Changing Environment conference proceedings, University of Salford, Salford, Education Development Unit. Retrieved from: http://www.ece.salford.ac.uk/proceedings/papers/ah_04.r tf

Kay, R.H.(2012). Exploring the use of video podcasts in education: a comprehensive review of the literature. Computers in Human Behavior, 28 (3), 820-831. 
Krug, S. (2013). Don't make me think!: a common sense approach to Web usability. New Riders; Edición: 3rd revised edition.

Letón, E., Durbán, M., D’Auria, B., \& Lee, D. J. (2009). Self learning mini videos through Internet and mobile telephones. Proceedings of EDULEARN09, 4277-4283.

Loranger, H. (2014). Break Grammar Rules on Websites for clarity. Retrieved from: http://www.nngroup.com/articles/break-grammar- rules/

MacLeod, L., Bergen, A., Storey, M.-A. (2017). Documenting and sharing software knowledge using screencasts Empirical Software Engineering, 22 (3), pp. 1478-1507. DOI: $10.1007 / \mathrm{s} 10664-017-9501-9$

Marketdata Enterprises (2011). Online education: An industry \& competitor analysis. Retrieved from: http://www.marketdataenterprises.com/FullIndustryStudi es.htm\#OnlineEducation

Pérez-Pérez, L., Andrés-Romero, M.P., Balsas-Almagro, J.R., Ruano-Ruano, I., Martín-Rodríguez, O., García-Cabrera,
L. (2011). A Proposal on E-learning Quality Assessment in Higher Education Initiative of Andalusian Virtual Campus. Edited by Pixel - International Conference The Future of Education. Florence, Italy 6-7 June 2011 Simonelli Editore - University Press.

Sánchez, A., Cancela, A., Maceiras, R., \& Urrejola, S. (2010). Multimedia productions: knowledge pills for university teaching. In IADIS International Conference e-Society, 351-355.

Traphagan, T., Kucsera, J.V., Kishi, K.K. (2010). Impact of class lecture webcasting on attendance and learning. Educational Technology Research and Development 58 (1), 19-37.

Valderrama, J.O., Sánchez, A. y Urrejola, S. (2009). International academic collaboration program on information technology and virtual teaching. Formación Universitaria. Retrieved from http://www.scielo.cl/scielo.php?pid=S071850062009000 $600002 \&$ script $=$ sci_arttext 